\title{
THE CONTROL OF SPECIFIC ACTUATORS FOR FAST FERRY VERTICAL MOTION DAMPING
}

\author{
Jose M. Giron-Sierra ${ }^{1}$, Reza Katebi ${ }^{2}$, Jesus M. de la Cruz ${ }^{1}$, Segundo Esteban ${ }^{1}$ \\ ${ }^{1}$ Dept. ACYA, Fac. Fisicas \\ Universidad Complutense de Madrid \\ 28040 Madrid, Spain \\ fax: (34) 91394 4687; e-mail: gironsi@dacya.ucm.es \\ ${ }^{2}$ Industrial Control Centre \\ Strathclyde University, UK
}

\begin{abstract}
The vertical motions of fast ships, which have a negative effect on comfort and safety, can be attenuated using moving actuators. There is the need of a control strategy to move the actuators in the most convenient way, considering several objectives. The paper considers this problem, studying a practical real example. Experiments with a scaled down replica of a fast ferry has been done, first for modeling purposes and second for control studies. Linear control shows serious application problems. Taking this into consideration, a nonlinear control approach is devised, with satisfactory results.
\end{abstract}

Index Terms - Ship Control, Marine Systems, Nonlinear Control, Multiobjective Optimization.

\section{INTRODUCTION}

High speed marine crafts are providing an important alternative for passenger transportation. To be competitive, speed is a crucial factor. Shipbuilders are applying new design concepts and advanced technologies to achieve fast ships, such the use of aluminum made hulls. At the same time, some problems take relevance. For instance, passenger's comfort. Perhaps some time ago seasickness was not considered a concern, but nowadays it can be a serious drawback for the possibly frequent passenger. In addition, the vertical (pitch and heave) motions of a fast ship may not be quite good for its structure and safety: in some sea conditions slamming and deck wetness occur.

As a matter of fact, the first trouble with ship motions appeared with the advent of motor ships: roll was not a problem with sailing. Roll motion was to be alleviated in the new era of naval transport. Several ideas were implemented, some in the form of appendages. Of special interest for our research is the pair of moving fins. This is an example of actuator, which motion must be controlled.

The case considered by our research is a fast ship equipped with two types of active appendages, devoted to alleviate pitch and heave motions (Fig. 1). One is a T-foil, under the hull and near the bow. The second is a pair of transom flaps. These actuators must move to counteract the effects of each incident wave [1] [2]. The problem is to find a good control of the actuators, to achieve comfort improvement under some application constraints.

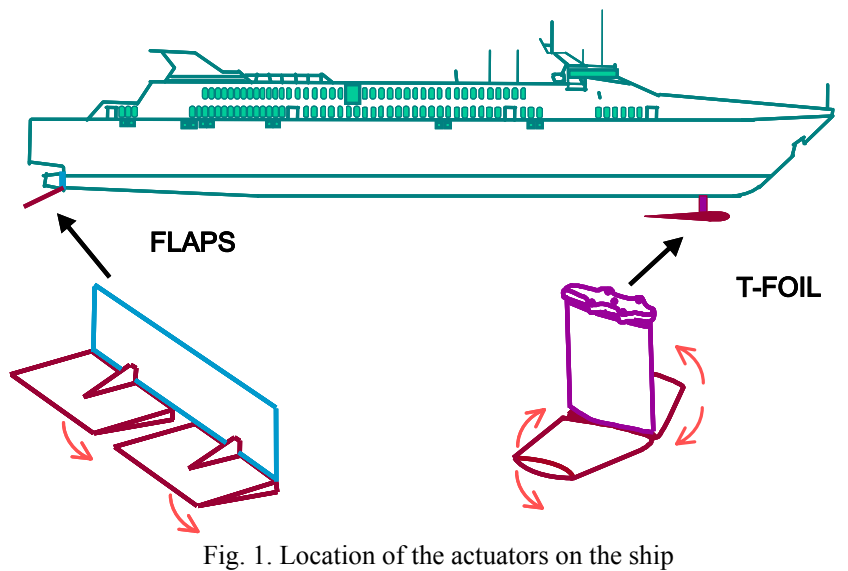

Two main steps were achieved along this research. The first was to obtain a realistic simulation environment, with facilities for fast and reliable control design studies. The second was to develop and test some initial control strategies. During this second step, nonlinear features were introduced in the control, with good results.

After a more detailed description of the problem, and some comments on the modeling experimental work, this paper focuses on the applied control aspects of our research. The paper concludes with experimental results.

\section{Characteristics Of The Problem}

\section{A. Ship Behavior}

Pitching and heaving motions are induced by waves. When the distance between successive waves is shorter than the ship's length, the ship lays always on two or more waves and the vertical motions are small. When this distance is longer than the ship's length, vertical motions can be large. Fig. 2 shows RAOS of pitch and heave motions at 20,30 and 40 knots (RAO is a relation of motion amplitude over wave amplitude, for several wavelengths). Notice resonance peaks, for wavelengths (Lambda) in the order of the ship's length (Lpp). 


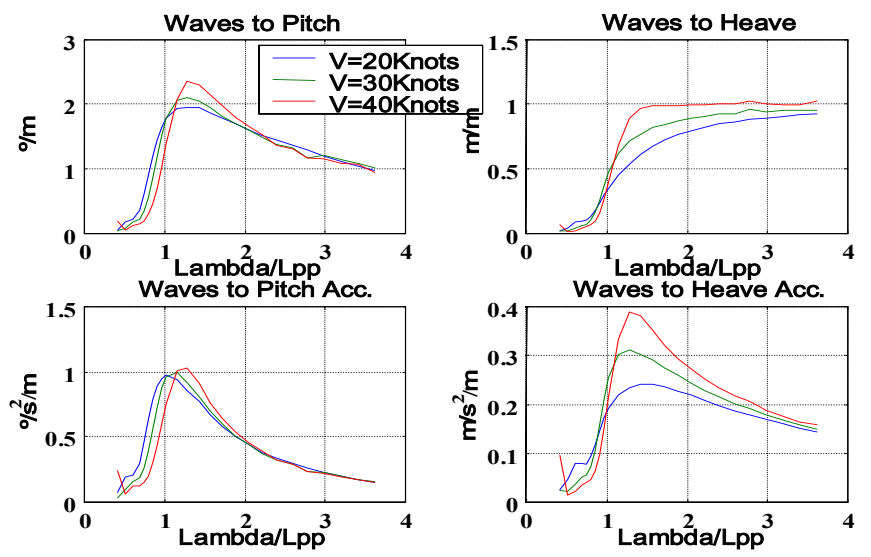

Fig. 2. RAOS of heaving and pitching motions

Suppose the ship is moving with head seas of regular waves with a fixed wavelength (this is really unusual, but it is an experiment that can be made in naval research facilities). As the ship's speed U increases, the frequency of encounter with waves also increases. If a frequency domain approach is considered for control design, on the basis of the frequency of encounter, some considerations are pertinent. Since pitching and heaving peaks (which could be related to poles) appear at certain fixed wavelengths, when these peaks are plotted with the frequency of encounter as the abscissa, these peaks must move as a function of $U$. In addition, the hydrodynamic characteristics of the ship (for instance, its added mass) change depending on $\mathrm{U}$. Considering all these factors, the mathematical models of the ship must change too.

Ocean waves are usually irregular. The World Meteorological Organization (WMO) distinguishes ten sea states, from SSN0 which is calm to SSN9 with very large waves [3]. SSN4, with a mean significant wave height of $1.875 \mathrm{~m}$, SSN5 with $3.25 \mathrm{~m}$, and SSN6 with $5.0 \mathrm{~m}$ are the sea states of interest for our research. In particular, SSN6 is rough enough to induce seasickness in many passengers and to run possible risks.

An important part of this research was devoted to establish satisfactory models of the ship's vertical motions. As will be described later on the paper, the result is a fairly complicated model, with several coupled transfer functions and some nonlinear blocks (related to actuators). In these conditions, an analytical tuning of controllers seems to be very difficult. In our case, an optimization procedure based on Genetic Algorithms was developed, to obtain a satisfactory control.

\section{B. Actuators Behavior}

The flaps and the T-foil can only exert limited actions [5]. For rough sea states, only a small fraction of vertical motions could be cancelled. However, for SSN4, which is a frequent state in many real operational scenarios (for instance, crossings in the Baltic Sea), the actuators can be very efficient. There are rate and angular limits for the motion of the actuators. In general, the actuators will reach frequently the limits, so there are saturations. Our experience with linear controllers is that optimization schemes lead to high gains, and this implies a bang-bang behavior of the actuators.

\section{Evaluation Indexes}

There is a mathematical model of seasickness, which predicts the percentage of passengers becoming sick after two hours of motions (this percentage is denoted as MSI: "Motion Sickness Incidence") [4]. Seasickness is due to the accumulated effect of vertical accelerations inside a frequency band around $0.16 \mathrm{~Hz}$. The model is the following (1), (2):

$$
M S I=100 \cdot\left[0.5 \pm \operatorname{erf}\left(\frac{ \pm \log _{10}\left(\overline{\xi_{3}} \mid / g\right) \mp \mu_{M S I}}{0.4}\right)\right]
$$

where $\overline{\left|\ddot{\mathrm{s}}_{3}\right|}$ is the r.m.s. vertical acceleration in a chosen place, and

$$
\mu_{M S I}=-0.819+2.32\left(\log _{10} \omega_{e}\right)^{2}
$$

where $w_{e}$ is the dominant frequency $(\mathrm{rads} / \mathrm{sec})$ of encounter with waves.

If the ship has a frequency of encounter with waves inside the band around $0.16 \mathrm{~Hz}$., a number of passengers will feel bad. This condition may happen (it depends on the ship's length) for certain combinations of sea spectra and ship's speeds.

The main control objective is to avoid seasickness. In addition, other objectives should also be considered:

- Vibration Index: Vibration of the actuators should be minimized. For economical and practical reasons (it is important to elude mechanical fatigue) it is not worth to move the actuators to counteract insignificant waves. Even with significant waves a smooth behavior is better.

- Cavitation Index: Cavitation eroding the actuators should be minimized. There is a limit attack angle which depends on speed: if this limit is surpassed cavitation appears.

Consequently, the problem calls for a multiobjective optimization of the control.

\section{DeVelopment Of A Simulation ToOL}

A fast ferry that is now in use for a link between Denmark and Norway is the research specific case. This ship is $110 \mathrm{~m}$ long, aluminum-made deep- $\mathrm{V}$ monohull, for 1,250 passengers, and able to reach 40 knots or more [6].

To establish mathematical models of the vertical motions [7], relevant data was obtained from two sources. One was a series of experiments with a scaled-down replica of the ship. The other was obtained using a CFD-based simulation program, which predicts forces, moments and motions of the ship, by 
means of fluid dynamics calculations and a geometric description of the hull.

Fig. 3 shows a photograph of the replica out of the water. The scale is $1 / 25$. The replica is equipped with a $T$-foil and a pair of transom flaps.

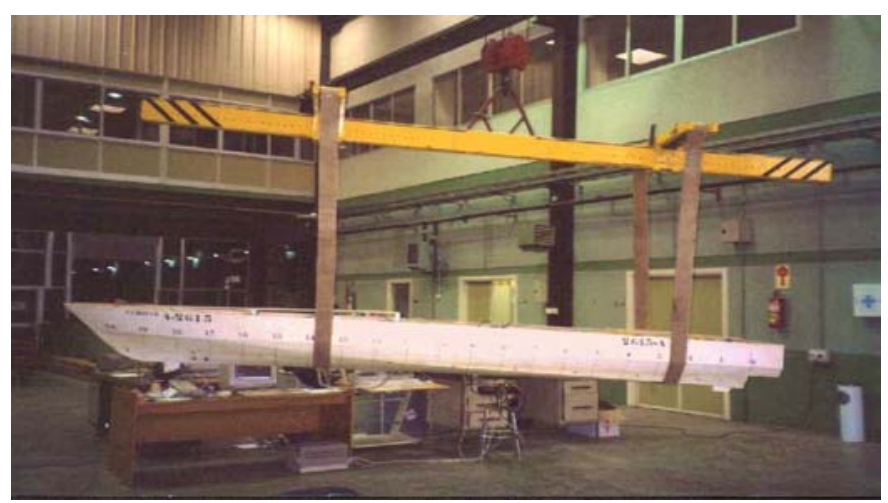

Fig. 3. Experimental scaled-down replica of the fast ferry

A series of experiments were achieved using the services of a Spanish towing tank institution (CEHIPAR, "Canal de Experiencias Hidrodinamicas de El Pardo", Madrid). The 300m long channel was used to obtain data of the actuators forces and moments, at several wings angles. The $100 \mathrm{~m} \times 30 \mathrm{~m}$ basin with wavemaker was used to gather data on the ship dynamic responses for 15 types of regular waves (15 different wavelengths), and also for irregular sea states SSN4, SSN5 and SSN6 with spectra corresponding to coastal waters (JONSWAP spectra). The experiments were run at speeds equivalent to 20 , 30 and 40 knots of the real ship.

The CFD-based program was also run for the same type of experiments (15 types of regular waves; 20, 30 and 40 knots). The most useful information given by the program concerns forces and moments. With this information a model of heaving and pitching motions was developed [8] using a decomposition: waves-to-forces and moments, forces and moments-to-motions. In addition, the effect of the actuators is easily integrated into the model, the forces and moments generated by actuators are added to the forces and moments generated by the waves. Fig. 4 illustrates the concept of the mathematical model and the connection of the actuators to the model.

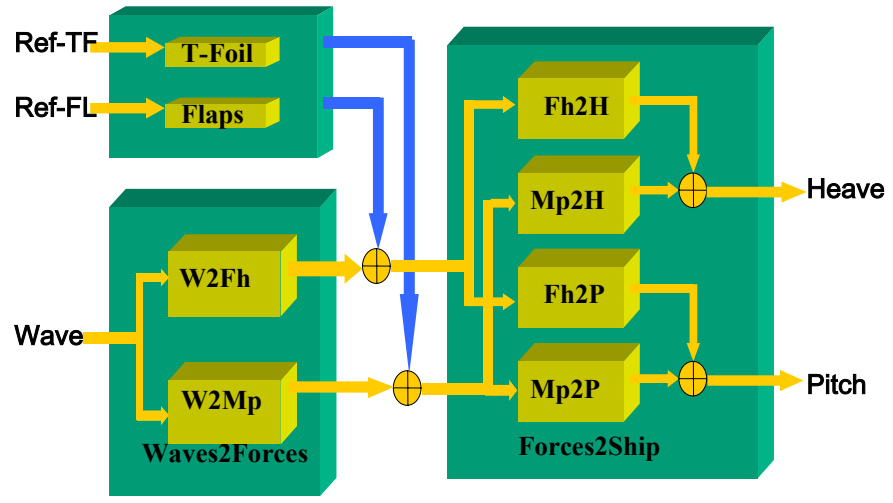

Fig. 4. Concept of the ship's vertical motions model
Each block in Fig. 4 represents a transfer function. Consequently, six transfer functions must be determined for each speed (20, 30 and 40 knots) and SSN (4, 5 and 6). A special identification method was developed, using genetic algorithms, to establish these transfer functions [9]. The dynamic behavior of the actuators was also modeled, based on physics first-principles and the analysis of the experimental data [10]. Non linear behaviors are contemplated in the models.

A complete SIMULINK model was developed, with provision of a block to be filled with a controller (to be designed and tested in this model). Fig. 5 shows the main toplevel view of the ship+actuators+control model.

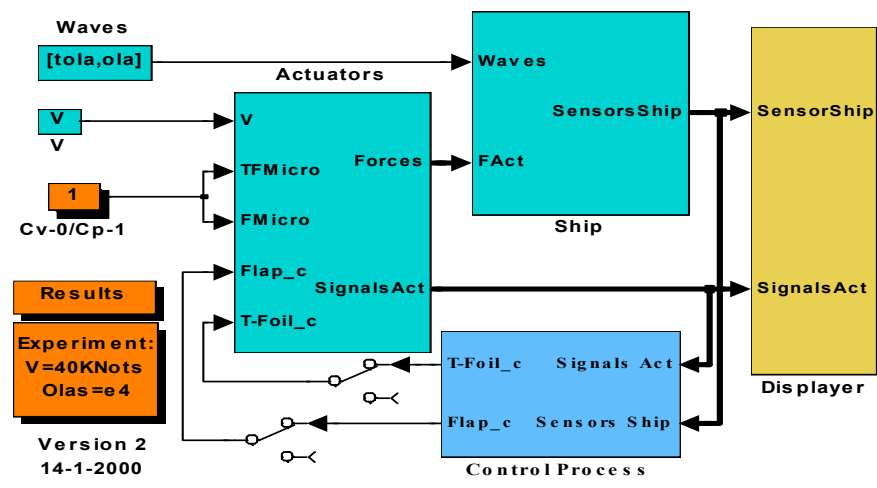

Fig. 5. Top-view of the SIMULINK model of the ship with actuators and control

Taking advantage of the GUI facilities offered by MATLAB-SIMULINK, a simulation environment was developed for control design studies [11], Fig. 6. The environment includes a set of standard experiments: the user can choose 20, 30 or 40 knots, and any of 15 wavelengths (for regular waves) or SSN4, 5 or 6 (for irregular waves). The objective of the simulation is to offer a platform for testing control strategies before any experiment at CEHIPAR (experiments have a cost). Since it is important to reproduce the experimental scenario, the noises registered by the sensors on the replica were recorded and integrated in the simulation.

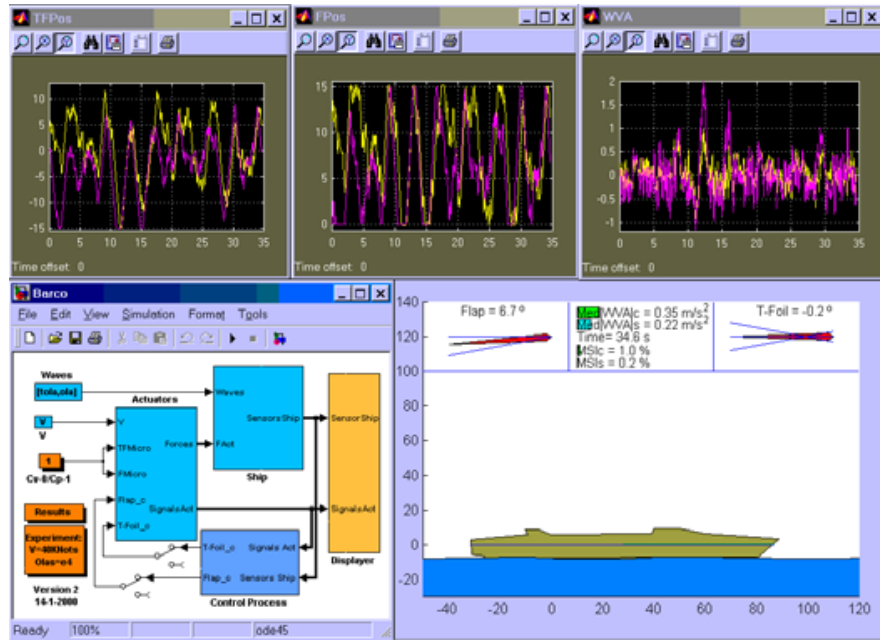

Fig. 6. Main screen of the simulation environment 
Fig. 6 shows the main screen of the simulation environment. The window at the left bottom of the screen is the SIMULINK complete model. The user can edit the SIMULINK block with the controller, to include a particular control into the simulation. The window at the right bottom is a moving figure of the ship, showing the motions of the actuators and the evolution of the MSI. The windows at the top display the evolution of the main variables of the control.

\section{EXPERIMENTAL PROBLEMS With CONTROL}

The experiments about control began with a conventional approach, to obtain a reference for comparison purposes: other control alternatives, to be investigated, should outperform this reference [13].

An accelerometer was installed in the worst place for passengers (near the bow) to measure what we denote as WVA ("Worst Vertical Acceleration"). Using the signal of the accelerometer as input, a PD was placed to control the flaps, and a second PD to control the T-foil. Using the simulation environment, it was noticed that any integral action of a PID was detrimental. Optimization of the PD parameters to minimize the MSI, which is similar to reduce the absolute mean of WVA, was obtained by a systematic search.

The instrumentation on board the replica is centered on an industrial Intel-based computer. The two PD controllers are digital, implemented in software. Through power electronics, the computer moves the flaps and the T-foil by means of step motors (1200 steps per revolution). Also, using signal conditioning electronic units, the computer takes data from the accelerometer (this is the input for the two PD controllers). The real-time control program was developed with a visual tool, EdRoom [12]. This environment automatically generates the code, so it was possible, in a quick and easy way, to introduce control improvements on the field, during experiments with the replica.

Initial tests of the replica with controlled actuators showed some real difficulties that are shown here:

\section{A. Noise}

One of the main problems is noise. There are several noise components in the spectrum of the experiments. Fig. 7 shows the spectrum and the temporal response of an experiment for a regular wave, $\mathrm{H}_{\mathrm{s}}=2.8 \mathrm{~m}$, with active control of the actuators. It has been necessary to run several experiments to determine each one of the spectrum components, the main components are:

1. Free response component, peak (a). The free response is very symmetric, and is placed at $2 \mathrm{~Hz}$.

2. Forced response component, peaks (a) and (b). Frequencies of (b) peaks are 2, 3 and 4 times the frequency of (a), this is due to the asymmetric response.

3. Vibration of the Hull. From 12 to $20 \mathrm{~Hz}$, peaks (c), there are vibrations that are propagated along the hull.
4. Vibration of the Actuators: the steps of the motors, the friction of the actuators (friction). First versions of the real time control induced brisk motion of the motors generating a lot of noise at 20 and $40 \mathrm{~Hz}$, peaks (d) and (e). This noise is more important in experiments with smaller waves.

5. Electromagnetic noise. This noise, at $50 \mathrm{~Hz}$, is not significant, except for small waves.
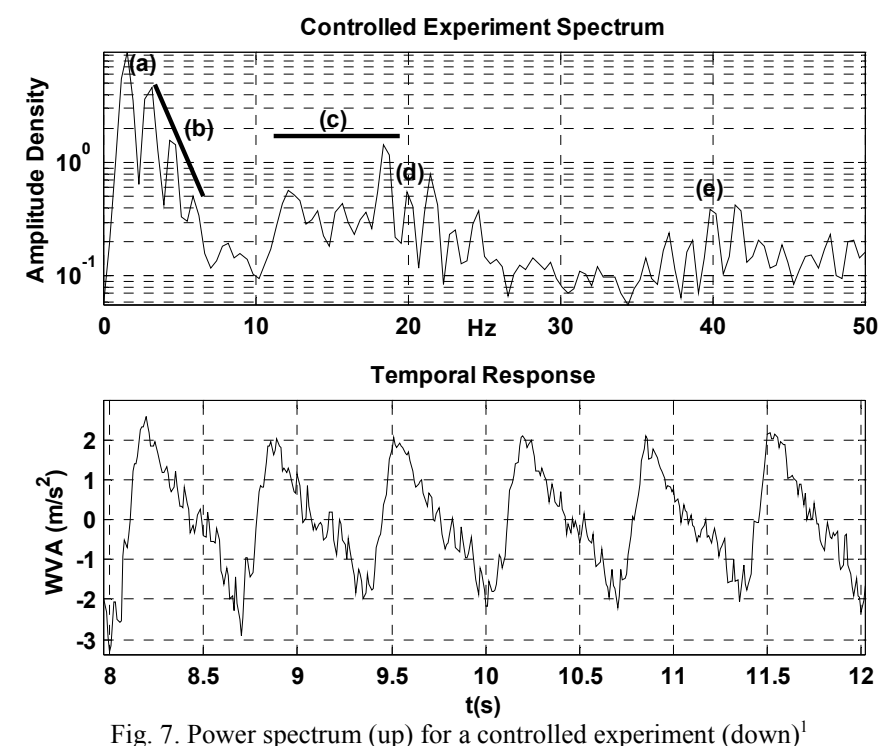

Since noise limits the derivative action we can exert with the $\mathrm{PD}$, a specific study was required to design a filter to eliminate the influence of noise in the control. Different alternatives of filter have been designed. Another problem is the asymmetry of the error signal, WVA, for example at the sec 8.5 changes the sign several times. It is possible that the actuators lost time changing their sense too, this is worse for the evaluation indexes. Then a good alternative is to filter (c) peaks and to ensure the unity gain at $2 \mathrm{~Hz}$ that is the main behavior of the ship. An example of discrete filter for a period of $10 \mathrm{~ms}$ is (3).

$$
\text { Noiseremover }(z)=\frac{0.1319 \cdot z^{2}-0.0026 \cdot z-0.1139}{z^{2}-1.7101 \cdot z+0.7304}
$$

\section{B. Bias}

Although the sensors are calibrated at the start of the experiments their behavior change along the time. The accelerometers accumulate a mean value that is not desired. There are to main reasons:

1. The temperature of the accelerometers changes along the day.

2. If the ship has a different trim angle in each experiment. The inclination of the accelerometer implies a nonzero mean value.

Since accelerations should have a zero mean value, a highpass filter must be designed to remove the bias. The simpler one is to compute the mean and to subtract it to the signal. An

\footnotetext{
${ }^{1}$ The scales of this figure are for the experimental replica.
} 
example that subtracts the mean in 6 seconds (The experiments in the towing tank have about 6 sec to stabilize the speed of the replica) for a sampling period of $10 \mathrm{~ms}$ seconds is presented in (4).

$$
\operatorname{Biasremover}(z)=\frac{200 \cdot z-200}{200 \cdot z-199}
$$

\section{TVR (Time Variant Relaxer)}

If the control has high gain, even small waves make the actuators to reach the angle limits. Thus an excessive control effort is invested for almost nothing (small waves have negligible effect on ship motions). Moving the actuators too much is also a source of vibrations, and a cause of mechanical fatigue. In addition, large angles of attack of the actuators originate cavitation. However, the optimization of control on the only basis of minimizing the MSI, leads to high gains [13].

To avoid the motion of the actuators for small accelerations, Fig. 8a and $8 \mathrm{~b}$ a dead zone can be inserted, Fig. 8c, so small vertical accelerations mean null error signal for the controller.
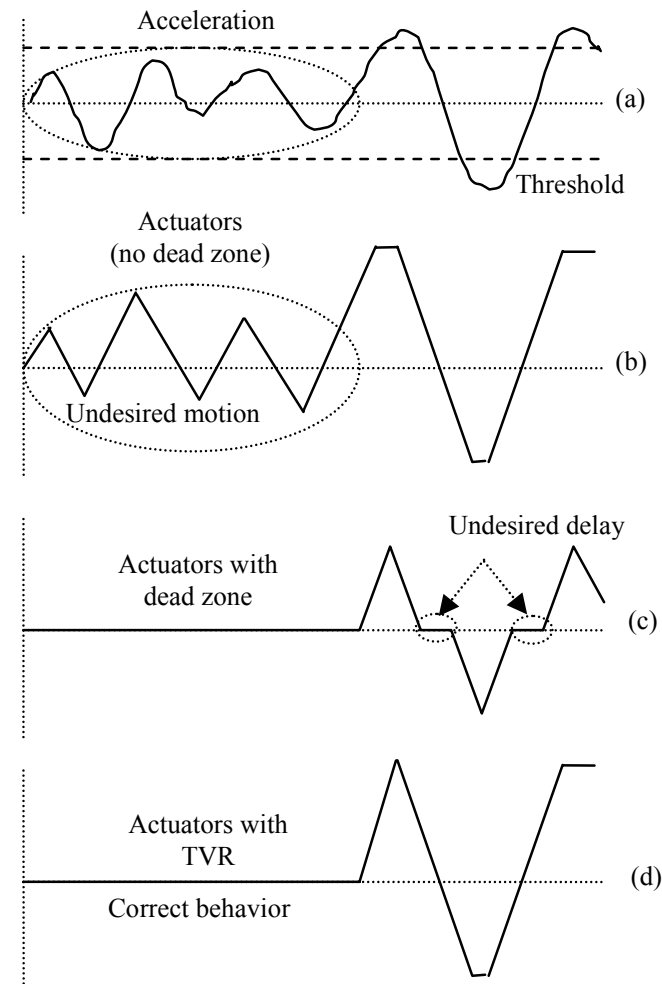

Fig. 8. Effect of a dead zone and TVR

It is clear that actuators must be synchronized to the incident waves. Delays are bad for this synchronization. Fig. 8c also shows that a collateral effect of the dead zone is an undesired delay. A new control strategy was devised to avoid this problem, the Time Variant Stabilizer. The desired behavior is shown in Fig. 8d. Let us suppose the input crosses the threshold of the dead zone, so actuators move. After some time $t_{\text {relax }}$ (measured from the threshold crossing) the actuators are forced to go to the zero position. This is accomplished according with a hyperbolic tangent trajectory of the control signal driving the actuators. Therefore, the control law is according to (5), (6) and (7):

$$
u_{a}= \begin{cases}u & \text { for } t_{\text {cross }}<t_{\text {relax }} \\ u_{r} & \text { for the rest }\end{cases}
$$

with

$$
u_{r}=\operatorname{Trim}+f_{\text {relax }}\left(t_{\text {cross }}\right) \cdot(u-\text { Trim })
$$

where Trim corresponds to the better static position of the actuators when there is no control, and

$$
f_{\text {relax }}\left(t_{\text {cross }}\right)=\frac{1}{2}\left(1+\text { hyp } \tan \left(3-6 \cdot\left(\frac{t_{\text {cross }}-t_{\text {relax }}}{t_{\text {relax }}}\right)\right)\right)
$$

In these expressions, $t_{\text {cross }}$ is measured from the threshold crossing, $u$ is the output of the PD and $u_{a}$ is the signal that drives the actuators. The next simulation, Fig. 9, shows the behavior of the TVR for an irregular wave. The experiment is a mixture of SSN4, SSN5 and SSN6.
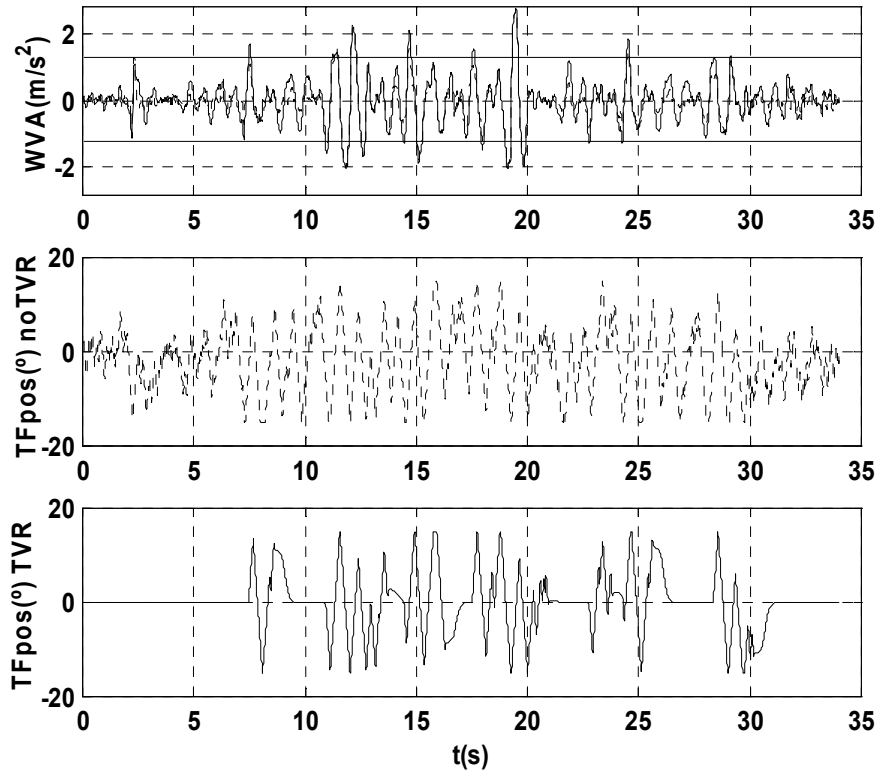

Fig.9. Effect of the TVR

In Fig. 9, the threshold is shown with two horizontal lines. If the filtered WVA surpasses the threshold, then the actuators must act. The WVA shown is the filtered WVA. Without TVR the T-Foil is moving all time, even when the WVA is very small. When TVR is active then $50 \%$ of the time the T-Foil is relaxed. Although the T-Foil has been relaxed, when there is a high WVA the T-Foil start and the WVA is similar to the case without TVR. The evaluation indexes of this experiment are presented in Fig. 10. In the first row there are no cavitation and no vibration because there is no control. Without TVR a very good MSI is obtained, but the Vibration and cavitation indexes increase too much. With the TVR, a good MSI is obtained at the same time that vibration and cavitation are reduced with respect to the no TVR case. 


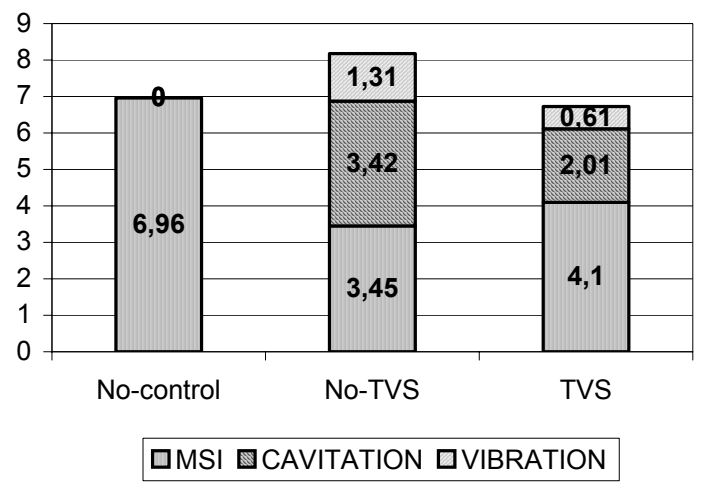

Fig.10. Improve with TVR.

\section{MultiobJECTIVE NON LiNEAR TUNING}

The filters of noise, the Bias remover, and the TVR are included in the Simulation Environment too. Then the tuning of the PD controllers will bear in mind these behaviors. It is very difficult to affront the nonlinearities of the actuators, the Time Variant Stabilizer, the non linear indexes, etc, in an analytical way; then optimization of simulations is the tuning method selected.

A multi-objective optimization with Genetic Algorithms was done combining the following targets:

- $\quad$ To minimize the MSI

- To minimize cavitation

- To minimize vibration of actuators

More over, doing the optimization for several conditions (Speed and SSN) at the same time it was possible to find a single tuning that is very good for all the conditions. Reference [14] explains it in detail.

\section{CONCLUSIONS}

The research deals with the control of flaps and a T-foil to improve the comfort of passengers, MSI, in a Fast-Ferry. These actuators must move to counteract the vertical motions induced by waves. It was noticed that not only the MSI (the Motion Sickness Incidence) need to be minimized, there are also other objectives to be considered by the control: less control efforts, less vibrations, less cavitation. Consequently, a multiobjective optimization problem has to be solved.

After a description of the characteristics of the problem, the main steps of the research were described in the paper. Experiments with conventional PD control showed several problems and some control strategies, like filters and TVR, were devised to solve them. The controller must be tuned bearing in mind the experimental problems and considering three main objectives at the same time. The results were clearly satisfactory.

\section{ACKNOWLEDGMENT}

The authors would like to thank the Spanish MCYT, "Ministerio de Ciencia y Tecnología", for their support (project DPI2000-0386-C03-02), the CEHIPAR staff for their cooperation, and IZAR for its recommendations.

\section{REFERENCES}

[1] M. Ryle, "Smoothing out the ride", The Motor Ship, January, pp. 23- 26, 1998.

[2] A.J. Haywood, A.J. Duncan, K.P. Klaka, and J. Bennet, "The development of a ride control system for fast ferries", Control Engineering Practice, pp. 695-703, 1995.

[3] A.R.J.M. Lloyd, Seakeeping: Ship Behavior in Rough Weather, A.R.J.M.Lloyd, Gosport, Hampshire, UK, 1998.

[4] J.F. O'Hanlon, and M.E. MacCawley, "Motion sickness incidence as a function of frequency and acceleration of vertical sinusoidal motion", Aerospace Medicine, 1974.

[5] E.V. Lewis, Principles of Naval Architecture, SNAME, New Jersey, 1989.

[6] Anonymous, "126 m long spanish fast ferry launched", Fast Ferries, September, pp. 19-20, 1996.

[7] T.J. Fossen, Guidance and Control of Ocean Vehicles, John Wiley, New York, 1994.

[8] S. Esteban, J.M. De la Cruz, J.M. Giron-Sierra, and B. De Andres, "Frequency-domain model of fast ferry vertical motions", in Proc. RINA Intl. Conf. Hydrodynamics of High Speed Craft, London, paper 18, pp. 1-8, 2000.

[9] B. De Andres, S. Esteban, J.M. Giron-Sierra and J.M. De la Cruz, "Modelling the motion of a fast ferry with the help of genetic algorithms", in Proc. IMACS Symp. on Mathematical Modelling, Viena, Austria, pp. 783-786, 2000.

[10] S. Esteban, J.M. De la Cruz, J.M. Giron-Sierra, B. De Andres, J.M. Diaz and J. Aranda, "Fast ferry vertical acceleration reduction with active flaps and T-foil", in Proc. IFAC Intl. Symp. on Maneuvering and Control of Marine Craft, Aalborg, Denmark, pp. 233-238, 2000.

[11] S. Esteban, B. De Andres, J.M. Giron-Sierra, O.R. Polo and E. Moyano, "A simulation tool for a fast ferry control design", in Proc. IFAC Intl. Conf. Control Applications in Marine Systems, Glasgow, U.K., 2001.

[12] O.R. Polo, S. Esteban, A. Maron, L. Grau and J.M. De la Cruz, "Control code generator used for control experiments in ship scale model", in Proc. IFAC Intl. Conf. Control Applications in Marine Systems, Glasgow, U.K., 2001.

[13] J.M. Giron-Sierra, S. Esteban, B. De Andres, J.M. Diaz and J.M. Riola, "Experimental study of controlled flaps and T-foil for comfort improvement of a fast ferry", in Proc. IFAC Intl. Conf. Control Applications in Marine Systems, Glasgow, U.K., 2001.

[14] S. Esteban, B. Andres-Toro, E. Besada-Portas, J.M. Giron-Sierra and J.M. de la Cruz, "Multiobjective control of flaps and T-foil in high-speed ships", accepted for the IFAC World Congress, Barcelona, Spain, 2002. 